\title{
Higher order self-dual models for spin-3 particles in $D=2+1$
}

\author{
D. Dalmazi, ${ }^{1, *}$ A. L. R. dos Santos, ${ }^{2, \dagger}$ and R. R. Lino dos Santos ${ }^{1, \hbar}$ \\ ${ }^{1}$ UNESP-Campus de Guaratinguetá-DFQ, CEP 12516-410, Guaratinguetá, São Paulo, Brazil \\ ${ }^{2}$ Instituto Tecnológico de Aeronáutica, DCTA, CEP 12228-900, \\ São José dos Campos, São Paulo, Brazil
}

(Received 3 August 2018; published 5 November 2018)

\begin{abstract}
In $D=2+1$ dimensions, elementary particles of a given helicity can be described by local Lagrangians (parity singlets). By means of a "soldering" procedure, two opposite helicities can be joined together and give rise to massive spin- $s$ particles carrying both helicities $\pm s$ (parity doublets), and such Lagrangians can also be used in $D=3+1$ to describe massive spin- $s$ particles. From this point of view the parity singlets (self-dual models) in $D=2+1$ are the building blocks of real massive elementary particles in $D=3+1$. In the three cases $s=1,3 / 2,2$ there are $2 s$ self-dual models of order $1,2, \ldots, 2 s$ in derivatives. In the spin-3 case the fifth order model is missing in the literature. Here we deduce a fifth order spin-3 self-dual model and fill up this gap. It is shown to be ghost free by means of a master action which relates it with the top model of sixth order. We believe that our approach can be generalized to arbitrary integer spin- $s$ in order to obtain the models of order $2 s$ and $2 s-1$. We also comment on the difficulties in relating the fifth order model with their lower order duals.
\end{abstract}

DOI: 10.1103/PhysRevD.98.105002

\section{INTRODUCTION}

Although we have not yet seen higher $\operatorname{spin}(s \geq 3 / 2)$ elementary particles in nature, massive particles of arbitrarily high spin are predicted by string theory. It is tempting to connect the nondetection of such particles to the theoretical difficulties we have in formulating a selfconsistent theory where such particles interact with themselves or with other fields like the gravitational field. As we increase the spin we need higher rank tensors. However, only $2 s+1$ degrees of freedom (d.o.f.) should survive for a massive spin-s particle in $D=3+1$ dimensions. Consequently, several spurious fields must be consistently eliminated which becomes cumbersome, especially when interactions are present. The tensor fields must obey the so-called Fierz-Pauli constraints [1].

Even the Lagrangians for free particles must be finetuned for higher spins in order to produce the correct constraints [2]. We believe that those Lagrangians could be systematically obtained from a "soldering" procedure of opposite helicities in $D=2+1$ space-time; see more on

\footnotetext{
*dalmazi@feg.unesp.br

†alessandroribeiros@yahoo.com.br

"rafa.robb@gmail.com
}

Published by the American Physical Society under the terms of the Creative Commons Attribution 4.0 International license. Further distribution of this work must maintain attribution to the author(s) and the published article's title, journal citation, and DOI. Funded by SCOAP ${ }^{3}$. soldering in [3-9]. In $D=2+1$ dimensions, differently from the real world, it is possible to write down local Lagrangians for elementary particles of given helicity. Helicity eigenstates are described by parity singlets, the so-called self-dual (SD) models in $D=2+1$. For instance, two Maxwell-Chern-Simons theories (spin-1) of helicities +1 and -1 , suggested in [10], can be soldered into the spin-1 Maxwell-Proca model whose action has the same form in arbitrary dimensions. Likewise, two spin-2 self-dual models of helicities +2 and -2 of second $\operatorname{order}^{1}$ in derivatives, suggested in [11], can be soldered into the massive spin-2 Fierz-Pauli (FP) theory. The fine-tuned mass term of the FP theory is automatically generated. It works also for higher derivative models. In particular, the relative $-3 / 8$ factor between $R_{\mu \nu^{-}}^{2}$ and $R^{2}$-terms in the linearized version of the "new massive gravity" (NMG) of [12] can also be automatically produced by the soldering of two linearized topologically massive gravities (LTMG) of opposite helicities +2 and -2 ; see [8]. The case of spin- $3 / 2$ has also been recently achieved [13]. The spin-3 case is still under investigation.

From a constructive point of view, we believe that the self-dual models in $D=2+1$ are the building blocks for massive particles in the real world. Therefore, it is certainly interesting to learn how to build them systematically for arbitrarily higher spins, especially their higher

\footnotetext{
${ }^{1}$ Throughout this work, $n$th order model stands for a Lagrangian whose maximal number of derivatives is $n$.
} 
order versions. As we increase the number of derivatives, the number of FP constraints which are identically satisfied as opposed to dynamically satisfied also increases which makes easier, in principle, the introduction of interactions without destroying the constraints.

The higher derivative self-dual models can be obtained from their first order counterparts order by order ${ }^{2}$ $\left(\mathcal{L}_{S D j}^{(s)} \rightarrow \mathcal{L}_{S D(j+1)}^{(s)}\right)$ in, at least, two different ways: either by means of a master action approach [14] (spin-1) (see $[15,16])$ for the spin-2 case, or via a Noether gauge embedding (NGE) procedure where the amount of local symmetries increases along with the number of derivatives; see [17] (spin-1), [18] (spin-3/2) and [16] (spin-2). Those three cases are consistent with a " $2 s$ " rule for the highest possible order in derivatives of a ghost-free selfdual model for spin-s particles. The case of spin-3 investigated here is quite challenging. Although the sixth order model is known [19], starting with the first order model of [20] we have obtained in [21] and [22] the second [23], the third [24], and a fourth order spin-3 selfdual model via the NGE and the master action procedures, respectively. Although they are all ghost-free, we have not been able to go beyond the fourth order and reach the sixth order model. The NGE procedure requires more symmetry in the higher order term than in the rest of the Lagrangian which is not the case in the $\mathcal{L}_{S D 4}^{(3)}$ model found in [21]. Regarding the master action, the highest order term in the Lagrangian cannot have any particle content which is not the case for either of $\mathcal{L}_{S D 4}^{(3)}$ as we have shown in [22]; see also Sec. IV in the present work. Here we tackle that problem by going downward from the sixth order theory [19] and finding a ghost-free fifth order model (Sec. II). In Sec. III we connect it with the sixth order model via master action. In Sec. IV we investigate a class of fourth order Lagrangians in search for a possible fourth order self-dual model different from $\mathcal{L}_{S D 4}^{(3)}$ of [22] which would allow us to go further downward from $\mathcal{L}_{S D 5}^{(3)}$. In Sec. V we have our conclusions and perspectives.

\section{THE FIFTH ORDER SELF-DUAL MODEL $\mathcal{L}_{S D 5}^{(3)}$}

We start this section recalling the construction of the sixth order spin-3 self-dual model $S D_{6}^{(3)}$ of [19]. We follow a route slightly different from [19] which we think could be more easily generalized to arbitrary integer spins. First, for the spin- 1 and spin- 2 cases, the highest self-dual models of order $2 s$ are given respectively by the MaxwellChern-Simons [10] and the linearized higher derivative topologically massive gravity of $[16,25]$. They can be

\footnotetext{
${ }^{2}$ The notation $\mathcal{L}_{S D n}^{(s)}$ stands for the Lagrangian density of the spin- $s$ self-dual model of order $n$ in derivatives.
}

written in a compact way with the help of dual fields $h^{*}$. Namely, ${ }^{3}$

$$
\begin{aligned}
\mathcal{L}_{S D 2}^{(1)} & =\frac{m}{2} h_{\mu} E^{\mu \nu} h_{\nu}^{*}-\frac{m^{2}}{2} h^{\mu} h_{\mu}^{*}, \\
\mathcal{L}_{S D 4}^{(2)} & =\frac{m}{2} h_{\mu}{ }^{\nu} E^{\mu \alpha} h_{\alpha \nu}^{*}-\frac{m^{2}}{2} h^{\mu \nu} h_{\mu \nu}^{*},
\end{aligned}
$$

where the dual fields are given by

$$
\begin{gathered}
h_{\mu}^{*}=E_{\mu \nu} h^{\nu} / m, \\
h_{\mu \nu}^{*}=\left(E_{\mu}{ }^{\alpha} \square \theta_{\nu}{ }^{\beta}+E_{\nu}{ }^{\alpha} \square \theta_{\mu}{ }^{\beta}\right) h_{\alpha \beta} /\left(2 m^{3}\right) .
\end{gathered}
$$

The transverse operators

$$
E^{\rho \delta} \equiv \epsilon^{\rho \delta \sigma} \partial_{\sigma} ; \quad \square \theta_{\rho \sigma} \equiv \square \eta_{\rho \sigma}-\partial_{\rho} \partial_{\sigma},
$$

are such that

$$
E^{\mu \nu} E^{\alpha \beta}=\square\left(\theta^{\mu \beta} \theta^{\nu \alpha}-\theta^{\mu \alpha} \theta^{\nu \beta}\right) .
$$

The dual fields identically satisfy the respective FP constraints:

$$
\partial^{\mu} h_{\mu}^{*}=0 ; \quad \partial^{\mu} h_{\mu \nu}^{*}=0 ; \quad \eta^{\mu \nu} h_{\mu \nu}^{*}=0 .
$$

The equations of motion are given, respectively, by

$$
\begin{aligned}
& E_{\mu}{ }^{\alpha} h_{\alpha}^{*}=m h_{\mu}^{*}, \\
& E_{(\mu}{ }^{\alpha} h_{\alpha \nu)}^{*}=2 m h_{\mu \nu}^{*} .
\end{aligned}
$$

By applying $E^{\beta \mu}$ on the equations of motion, using (5) and (6), equations of motion recursively and symmetrizing the result (only in the spin-2 case of course) we derive the Klein-Gordon (KG) equations:

$$
\left(\square-m^{2}\right) h_{\beta}^{*}=0 ; \quad\left(\square-m^{2}\right) h_{\beta \nu}^{*}=0 .
$$

The Pauli-Lubanski equations, (7) and (8), single out one helicity eigenstate. They form altogether with (6) and (9) all the required equations for helicity-s particles $(s=1,2)$ in $D=2+1$ dimensions (parity singlets) represented by the dual field $h^{*}$. The natural generalization of (1) for spin-3 would be a sixth order self-dual model for a totally symmetric rank-3 tensor,

$$
\mathcal{L}_{S D 6}^{(3)}=\frac{m}{2} h_{\mu}{ }^{\nu \rho} E^{\mu \alpha} h_{\alpha \nu \rho}^{*}-\frac{m^{2}}{2} h^{\mu \nu \rho} h_{\mu \nu \rho}^{*} .
$$

\footnotetext{
${ }^{3}$ We use $\eta_{\mu \nu}=(-,+,+),(\alpha \beta)=\alpha \beta+\beta \alpha$ and $(\alpha \beta \gamma)=\alpha \beta \gamma+$ $\beta \gamma \alpha+\gamma \alpha \beta$.
} 
The totally symmetric dual field $h_{\mu \nu \rho}^{*}$ is obtained by applying some fifth order differential operator on $h_{\mu \nu \rho}$ such that the required spin-3 Fierz-Pauli constraints are identically satisfied:

$$
\partial^{\mu} h_{\mu \nu \rho}^{*}=0 ; \quad \eta^{\mu \nu} h_{\mu \nu \rho}^{*}=0 .
$$

If we apply $E_{\beta}{ }^{\mu}$ on the equations of motion:

$$
E_{(\mu}{ }^{\alpha} h_{\alpha \nu \rho)}^{*}=3 m h_{\mu \nu \rho}^{*},
$$

and use (5), (11), and (12) recursively, we obtain the expected KG equations $\left(\square-m^{2}\right) h_{\beta \nu \rho}^{*}=0$.

The invariance of the third order Lagrangian $h^{\mu \nu} h_{\mu \nu}^{*}$ under linearized reparametrizations and Weyl transformations $\delta h_{\mu \nu}=\partial_{(\mu} \Lambda_{\nu)}+\eta_{\mu \nu} \psi$ is enough to guarantee that the spin-2 FP constraints in (6) hold identically. Likewise, we require invariance of the fifth order Lagrangian $h^{\mu \nu \rho} h_{\mu \nu \rho}^{*}$ under

$$
\delta h_{\mu \nu \rho}=\partial_{(\mu} \Lambda_{\nu \rho)}+\eta_{(\mu \nu} \psi_{\rho)} .
$$

The first symmetry implies that the six indices of the two $h_{\mu \nu \rho}$ fields present in $h^{\mu \nu \rho} h_{\mu \nu \rho}^{*}$ be contracted with indices of transverse operators $E^{\mu \nu}$ or $\square \theta^{\mu \nu}$. There are only two possibilities at fifth order:

$$
\mathcal{L}_{A B}=h^{\mu \nu \rho} \square^{2} E_{\mu}{ }^{\alpha}\left(A \theta_{\nu}^{\beta} \theta_{\rho}^{\gamma}+B \theta_{\nu \rho} \theta^{\beta \gamma}\right) h_{\alpha \beta \gamma}
$$

The vector Weyl invariance then fixes $B=-A / 4$. Therefore, the sixth order spin-3 self-dual model, in agreement with [19], is given by (10) where

$$
h_{\mu \nu \rho}^{*}=\frac{\square^{2}}{3 m^{5}} E_{(\mu}^{\alpha}\left(\theta_{\nu}^{\beta} \theta_{\rho)}^{\gamma}-\frac{1}{4} \theta_{\nu \rho)} \theta^{\beta \gamma}\right) h_{\alpha \beta \gamma} .
$$

Alternatively, one can start with a rather general ansatz for a fifth order Lagrangian including all possible contractions (five terms) and the symmetry (13) will finally lead us to the same answer.

Both sixth and fifth order terms in $\mathcal{L}_{S D 6}^{(3)}$ are invariant under the same set of gauge transformations (13). This is the typical situation in the highest $(2 s)$ order self-dual models; see [16]. The high degree of symmetry avoids the presence of ghosts which commonly appear in higher derivative theories. The absence of ghosts in $\mathcal{L}_{S D 6}^{(3)}$ has been explicitly proven in [19] in the gauge $\partial_{j} h_{j \mu \nu}=0$. They have also shown that the fifth order term $h^{\mu \nu \rho} h_{\mu \nu \rho}^{*}$ has by itself no particle content, very much like the third order linearized gravitational Chern-Simons term in the spin-2 case; see [10]. Since the latter one can be combined with the linearized Einstein-Hilbert action (another empty theory) in order to produce a meaningful spin-2 self-dual model (linearized topologically massive gravity), one naturally wonders whether we could combine $h^{\mu \nu \rho} h_{\mu \nu \rho}^{*}$ with some fourth order term and end up with a ghost free fifth order self-dual model.

As we have already mentioned, there is a systematic procedure to go from the $n$th to the $(n+1)$ th order self-dual model by either using the embedding of gauge symmetries or a master action [14] approach. However, to the best we know there is no systematic way to go downward in derivatives. In the spin-2 and spin-1 cases both self-dual models of order $2 s-1$, i.e., $\mathcal{L}_{S D 3}^{(2)}$ and $\mathcal{L}_{S D 1}^{(1)}$, are built up from two terms of zero particle content. This is a key ingredient in the master action approach. So we must seek for a highly symmetric fourth order term. Starting with a general ansatz:

$$
\begin{aligned}
\mathcal{L}^{(4)}= & a h_{\mu \nu \alpha} \square^{2} h^{\mu \nu \alpha}+b h_{\mu} \square^{2} h^{\mu}+c h_{\mu \nu \alpha} \square \partial^{\mu} \partial_{\rho} h^{\rho \nu \alpha} \\
& +d h_{\mu} \square \partial^{\mu} \partial_{\nu} h^{\nu}+e h_{\mu \nu \alpha} \partial^{\mu} \partial^{\nu} \square h^{\alpha} \\
& +f \partial^{\mu} \partial^{\nu} h_{\mu \nu \alpha} \partial_{\gamma} \partial_{\rho} h^{\gamma \rho \alpha}+g \partial^{\mu} \partial^{\nu} \partial^{\alpha} h_{\mu \nu \alpha} \partial_{\rho} h^{\rho} .
\end{aligned}
$$

Let us first define a subclass of models invariant under traceless reparametrizations ${ }^{4}$ :

$$
\delta h_{\mu \nu \rho}=\partial_{(\mu} \bar{\Lambda}_{\nu \rho)} ; \quad \eta^{\mu \nu} \bar{\Lambda}_{\mu \nu}=0 .
$$

Such symmetry can be implemented if

$c=-3 a ; \quad d=\frac{1}{4}(9 a+5 b) ; \quad e=-2 b ;$

$f=g=3 a+b$.

Accordingly, (16) becomes

$$
\mathcal{L}_{(a, b)}^{(4)}=a\left(\mathbb{R}_{\mu \nu \rho} \mathbb{R}^{\mu \nu \alpha}-\frac{3}{4} \mathbb{R}_{\mu} \mathbb{R}^{\mu}\right)+\frac{b}{4} \mathbb{R}_{\mu} \mathbb{R}^{\mu},
$$

where the spin-3 Ricci-like curvature and its vector contraction have been introduced in [24], namely,

$$
\begin{aligned}
\mathbb{R}_{\mu \nu \rho}= & \square h_{\mu \nu \rho}-\partial^{\alpha}\left(\partial_{\mu} h_{\alpha \nu \rho}+\partial_{\nu} h_{\alpha \mu \rho}+\partial_{\rho} h_{\alpha \mu \nu}\right)+\partial_{\mu} \partial_{\nu} h_{\rho} \\
& +\partial_{\nu} \partial_{\rho} h_{\nu}+\partial_{\rho} \partial_{\mu} h_{\nu}, \\
\mathbb{R}_{\rho}= & \eta^{\mu \nu} \mathbb{R}_{\mu \nu \rho}=2\left(\square h_{\rho}-\partial^{\alpha} \partial^{\beta} h_{\alpha \beta \rho}+\frac{1}{2} \partial^{\alpha} \partial_{\rho} h_{\alpha}\right) .
\end{aligned}
$$

Note that $\mathbb{R}_{\mu \nu \rho}$, and consequently $\mathbb{R}_{\mu}$, is invariant under (17). The traceless reparametrization symmetry (17) plays in the massive spin-3 theory in $D=2+1$, and also in $D=3+1$ [2], the same role of the linearized reparametrizations

\footnotetext{
${ }^{4}$ There is one case where we have invariance under arbitrary reparametrizations $\delta h_{\mu \nu \alpha}=\partial_{(\mu} \lambda_{\nu \alpha)}$; however, it coincides with the fourth order term appearing in [21] which contains a ghost.
} 
$\delta h_{\mu \nu}=\partial_{(\mu} \Lambda_{\nu)}$ in the spin-2 FP theory, i.e., it is the symmetry of the massless limit of the theory and is instrumental in deriving the FP constraints in the massive case.

Now we are ready to suggest an ansatz for the fifth order spin-3 self-dual model. Usually, the lower derivative term of the $n$th order self-dual model becomes the higher derivative term of the $(n-1)$ th model but with opposite sign. Thus, the second term of (10), with opposite sign, now becomes the highest order term of $\mathcal{L}_{S D 5}^{(3)}$. We add up the fourth order term (19) and require the final Lagrangian to fit into the form (10) in terms of a fourth order dual field $\tau_{\mu \nu \rho}^{*}$ :

$\mathcal{L}_{S D 5}^{(3)}=\frac{m^{2}}{2} h^{\mu \nu \rho} h_{\mu \nu \rho}^{*}-\mathcal{L}_{(a, b)}^{(4)}=\frac{m}{2} h_{\mu}{ }^{\nu \rho} E^{\mu \alpha} \tau_{\alpha \nu \rho}^{*}-\frac{m^{2}}{2} h^{\mu \nu \rho} \tau_{\mu \nu \rho}^{*}$.

From (21) and (15) we end up with a unique solution:

$$
a=\frac{1}{2 m^{2}} ; \quad b=-\frac{3}{4} a=-\frac{3}{8 m^{2}} .
$$

Explicitly, the fourth order dual field is given by

$$
\begin{aligned}
\tau_{\mu \nu \rho}^{*}= & \frac{1}{m^{4}}\left[\square^{2} h_{\mu \nu \rho}-\frac{1}{4} \eta_{(\mu \nu} \square^{2} h_{\rho)}-\square \partial_{(\mu} \partial^{\beta} h_{\beta \nu \rho)}\right. \\
& +\frac{\square}{4} \eta_{(\mu \nu} \partial_{\gamma} \partial_{\beta} h_{\rho)}^{\gamma \beta}+\frac{\square}{4} \partial_{(\mu} \partial_{\nu} h_{\rho)}+\frac{7}{16} \eta_{(\mu \nu} \partial_{\rho)} \square \partial \cdot h \\
& +\frac{3}{4} \partial_{(\mu} \partial_{\nu} \partial^{\gamma} \partial^{\beta} h_{\gamma \beta \rho)}-\frac{9}{8} \partial_{\mu} \partial_{\nu} \partial_{\rho}(\partial \cdot h) \\
& \left.-\frac{3}{8} \eta_{(\mu \nu} \partial_{\rho)} \partial^{\gamma} \partial^{\beta} \partial^{\delta} h_{\gamma \beta \delta}\right] .
\end{aligned}
$$

It turns out that the fourth order term in (21) is exactly the same one appearing in the fourth order description of massive spin-3 particles (parity doublet) of [26]. It can be written as the product of a spin-3 second order Einstein-like and a Schouten-like tensor very much like the fourth order term (K-term) of the NMG theory [12]. After integrating by parts we can write

$$
\begin{aligned}
\mathcal{L}_{S G} & \equiv \frac{m^{2}}{2} h^{\mu \nu \rho} \tau_{\mu \nu \rho}^{*}=\frac{1}{2 m^{2}} \mathbb{S}_{\mu \nu \alpha}(h) \mathbb{G}^{\mu \nu \alpha}(h) \\
& =\frac{1}{2 m^{2}} \mathbb{R}_{\mu \nu \lambda} \mathbb{R}^{\mu \nu \lambda}-\frac{15}{32 m^{2}} \mathbb{R}_{\mu} \mathbb{R}^{\mu},
\end{aligned}
$$

where (see [24])

$$
\begin{aligned}
& \mathbb{G}_{\mu \nu \lambda} \equiv \mathbb{R}_{\mu \nu \lambda}-\frac{1}{2} \eta_{(\mu \nu} \mathbb{R}_{\lambda)} ; \\
& \mathbb{S}_{\mu \nu \lambda}=\mathbb{G}_{\mu \nu \lambda}-\frac{1}{4} \eta_{(\mu \nu} \mathbb{G}_{\lambda)}=\mathbb{R}_{\mu \nu \lambda}-\frac{1}{8} \eta_{(\mu \nu} \mathbb{R}_{\lambda)} .
\end{aligned}
$$

The definition of the Einstein-like tensor is consistent with the traceless reparametrization in $D=2+1$ in the sense that $\mathbb{G}_{\mu \nu \lambda}(h)=0$ implies a pure gauge solution $h_{\mu \nu \rho}=$ $\partial_{(\mu} \bar{\Lambda}_{\nu \rho)}$ just like $G_{\mu \nu}(h)=0$ leads to $h_{\mu \nu}=\partial_{(\mu} \Lambda_{\nu)}$ in the spin-2 case. Besides traceless reparametrizations (17), the fourth order term $\mathcal{L}_{S G}$ is the only possible combination among all fourth order terms (16) which is invariant also under transverse Weyl transformation (quite similar again to the K-term in NMG)

$$
\delta h_{\mu \nu \rho}=\eta_{(\mu \nu} \psi_{\rho)}^{T} ; \quad \partial^{\mu} \psi_{\mu}^{T}=0 .
$$

The fifth order term in (21) can also be written in a more inspiring form, namely,

$$
\frac{m^{2}}{2} h^{\mu \nu \rho} h_{\mu \nu \rho}^{*}=\frac{1}{4 m^{3}} \mathbb{S}_{\mu \nu \alpha}(h) \mathbb{G}^{\mu \nu \alpha}(E h),
$$

where $(E h)_{\mu \nu \alpha}=(2 / 3) E_{(\mu \beta} h_{\nu \alpha)}^{\beta}$. Notice that the fifth order term in $\mathcal{L}_{S D 5}^{(3)}$ is invariant under (17) and full Weyl transformations; see (13). It is a common feature of lower order (below $2 s$ ) self-dual models that the highest derivative term has more symmetries than its lower derivative partner. Next we show via master action that the particle content of $\mathcal{L}_{S D 5}^{(3)}$ is the same one of $\mathcal{L}_{S D 6}^{(3)}$, i.e., helicity +3 or -3 particles, depending on the sign of the fifth order term, without ghosts.

The generalization of the sixth order model (10) for arbitrary spin- $s$ goes in the following way. We first replace $h_{\mu \nu \rho}$ by a rank-s totally symmetric field $h_{\alpha_{1} \cdots \alpha_{s}}$. The dual field $h_{\alpha_{1} \cdots \alpha_{s}}^{*}$ is built up out of a differential operator of order $2 s-1$ applied on $h_{\alpha_{1} \cdots \alpha_{s}}$ such that the rank-s generalization of (13) becomes a symmetry of $h^{\alpha_{1} \cdots \alpha_{s}} h_{\alpha_{1} \cdots \alpha_{s}}^{*}$. As we increase the spin we have more terms which contribute; however, the rank-s version of (13) is enough [27] to uniquely fix the dual field $h_{\alpha_{1} \cdots \alpha_{s}}^{*}$. The factor $3 m$ will be replaced by $s m$ on the right-hand side of (12). We believe that the lower order $2 s-1$ self-dual model can be defined for arbitrary spin-s following the same route of the fifth order spin-3 model. Namely, we define a term of order $2 s-2$ by requiring traceless reparametrizations and then impose that its symmetrized curl becomes the already known $2 s-1$-term $h^{\alpha_{1} \cdots \alpha_{s}} h_{\alpha_{1} \cdots \alpha_{s}}^{*}$. This is in progress [27]. The increasing number of derivatives does not lead to ghosts due to the increasing number of local symmetries. The proof of absence of ghosts is still cumbersome due to the higher derivatives.

\section{MASTER ACTION: $S_{\text {SD5 }}^{(3)} \rightarrow S_{\text {SD6 }}^{(3)}$}

In this section we use the master action technique to connect $\mathcal{L}_{S D 5}^{(3)}$ with $\mathcal{L}_{S D 6}^{(3)}$. We start with the $S_{S D 5}$ action and 
add a mixing term between the old field and the new dual field. The mixing term is the fifth order term of $S_{S D 5}$.

The SD5 action is given by

$$
\begin{aligned}
S_{S D 5}^{(3)}[h]= & \int d^{3} x\left[-\frac{1}{2 m^{2}} \mathbb{S}_{\mu \nu \alpha}(h) \mathbb{G}^{\mu \nu \alpha}(h)\right. \\
& \left.+\frac{1}{4 m^{3}} \mathbb{S}_{\mu \nu \alpha}(h) \mathbb{G}^{\mu \nu \alpha}(E h)\right] .
\end{aligned}
$$

It has been shown in [19] that the fifth order term has no particle content. The fourth order term $\mathcal{L}_{S G}$ has also an empty spectrum as we have shown in [26] via a duality transformation to a rank-2 theory; we will say more about it later on. Following the master action procedure, we use the fifth order term as mixing to construct the following master action:

$S_{M}[h, f]=S_{S D 5}^{(3)}[h]-\frac{1}{4 m^{3}} \int d^{3} x \mathbb{S}_{\mu \nu \alpha}(h-f) \mathbb{G}^{\mu \nu \alpha}(E(h-f))$,

where $f_{\mu \nu \alpha}$ is the new field introduced through the mixing term. In order to find a dual map between correlation functions of the dual models, we add a source term $j^{\mu \nu \alpha}$ coupled to a totally symmetric dual field $\tau_{\mu \nu \alpha}^{*}$ and define the following generating functional:

$$
Z_{M}[j]=\int \mathcal{D} h \mathcal{D} f \exp i\left(S_{M}[h, f]+\int d^{3} x \tau_{\mu \nu \alpha}^{*} j^{\mu \nu \alpha}\right) .
$$

The dual field is given by $\tau_{\mu \nu \alpha}^{*}=\left(1 / m^{4}\right) \mathbb{G}_{\mu \nu \alpha}(\mathbb{S}(h))$; see explicitly in (23). It guarantees the gauge symmetry of the master action under $\delta h_{\mu \nu \alpha}=\partial_{(\mu} \bar{\Lambda}_{\nu \alpha)}+\eta_{(\mu \nu} \psi_{\alpha)}^{T}$.

After making the shift $f \rightarrow f+h$ in (29) we recover the fifth order self-dual model plus a decoupled fifth order term depending only on $f_{\mu \nu \rho}$. Since the fifth order term has no particle content, this guarantees that the particle content of $S_{M}[h, f]$ is the same of $S_{S D 5}^{(3)}[h]$. On the other hand, the action can be written as ${ }^{5}$

$$
\begin{aligned}
S_{M}= & \int d^{3} x\left[-\frac{1}{4 m^{3}} \mathbb{S}_{\mu \nu \alpha}(f) \mathbb{G}^{\mu \nu \alpha}(E f)-\frac{1}{2 m^{2}} \mathbb{S}_{\mu \nu \alpha}(h) \mathbb{G}^{\mu \nu \alpha}(h)\right. \\
& \left.+\frac{1}{2 m^{3}} \mathbb{S}_{\mu \nu \alpha}(h) \mathbb{G}^{\mu \nu \alpha}(E f)+\frac{1}{m^{4}} \mathbb{S}_{\mu \nu \alpha}(h) \mathbb{G}^{\mu \nu \alpha}(j)\right], \quad \text { (31) }
\end{aligned}
$$

\footnotetext{
${ }^{5}$ We have used the following properties in the source term: $\mathbb{G}_{\mu \nu \alpha}(\mathbb{S}(h)) j^{\mu \nu \alpha}=\mathbb{G}_{\mu \nu \alpha}(h) \mathbb{S}^{\mu \nu \alpha}(j)=\mathbb{S}_{\mu \nu \alpha}(h) \mathbb{G}^{\mu \nu \alpha}(j)$.
}

which is equivalent to

$$
\begin{aligned}
S_{M}= & \int d^{3} x\left[-\frac{1}{4 m^{3}} \mathbb{S}_{\mu \nu \alpha}(f) \mathbb{G}^{\mu \nu \alpha}(E f)\right. \\
& +\frac{1}{8 m^{4}} \mathbb{S}_{\mu \nu \alpha}(E f) \mathbb{G}^{\mu \nu \alpha}(E f)+\frac{1}{4 m^{5}} \mathbb{G}_{\mu \nu \alpha}(E f) \mathbb{S}^{\mu \nu \alpha}(j) \\
& \left.-\frac{1}{2 m^{2}} \mathbb{S}_{\mu \nu \alpha}\left(h-\frac{E f}{2 m}-\frac{j}{m^{2}}\right) \mathbb{G}^{\mu \nu \alpha}\left(h-\frac{E f}{2 m}-\frac{j}{m^{2}}\right)\right] .
\end{aligned}
$$

After making the shift

$$
h_{\mu \nu \alpha} \rightarrow h_{\mu \nu \alpha}+\frac{1}{2 m}(E f)_{\mu \nu \alpha}+\frac{j_{\mu \nu \alpha}}{m^{2}},
$$

the last term of (32) decouples. Since such a term has no particle content it can be trivially Gaussian integrated. Thus, we finally obtain the sixth order self-dual model given by ${ }^{6}$

$$
\begin{aligned}
S_{S D 6}^{(3)}[f]= & \int d^{3} x\left[-\frac{1}{4 m^{3}} \mathbb{S}_{\mu \nu \alpha}(f) \mathbb{G}^{\mu \nu \alpha}(E f)\right. \\
& \left.+\frac{1}{8 m^{4}} \mathbb{S}_{\mu \nu \alpha}(f) \mathbb{G}^{\mu \nu \alpha}\left(E^{2} f\right)+f_{\mu \nu \alpha}^{*} j^{\mu \nu \alpha}+\mathcal{O}\left(j^{2}\right)\right],
\end{aligned}
$$

where $f_{\mu \nu \alpha}^{*}=\left(1 / 2 m^{5}\right) \mathbb{G}_{\mu \nu \alpha}(\mathbb{S}(E f))$ is the dual field $h_{\mu \nu \alpha}^{*}$ given in (15) after the replacement $h_{\mu \nu \alpha} \rightarrow f_{\mu \nu \alpha}$. The equivalence between the $S_{S D 5}^{(3)}$ and $S_{S D 6}^{(3)}$ actions is guaranteed by the master action $S_{M}$. So the action (34) describes helicity +3 (or -3 ) eigenmodes depending on the sign of the fifth order term. The correlation functions in both theories are related by

$\left\langle\tau_{\mu_{1} \nu_{1} \alpha_{1}}^{*} \ldots \tau_{\mu_{N} \nu_{N} \alpha_{N}}^{*}\right\rangle_{S D 5}=\left\langle f_{\mu_{1} \nu_{1} \alpha_{1}}^{*} \ldots f_{\mu_{N} \nu_{N} \alpha_{N}}^{*}\right\rangle_{S D 6}+$ C.T.,

where C.T. are contact terms which appear due to the quadratic terms on the sources in the master action. The equations of motion of $\mathcal{L}_{S D 5}^{(3)}(h)$ are mapped in the equations of motion of $\mathcal{L}_{S D 6}^{(3)}(f)$ via the substitution

$$
\tau_{\mu \nu \alpha}^{*} \rightarrow f_{\mu \nu \alpha}^{*} .
$$

The equivalence between the new fifth order spin-3 selfdual model given in (21) or (28) and the sixth order model of [19] raises the question of whether there might be another master action allowing us to go further downwards to reach a fourth order self-dual model and eventually fill

\footnotetext{
${ }^{6} \mathrm{We}$ have used the property: $\mathbb{S}_{\mu \nu \alpha}(E f) \mathbb{G}^{\mu \nu \alpha}(E f)=$ $\mathbb{S}_{\mu \nu \alpha}(f) \mathbb{G}^{\mu \nu \alpha}\left(E^{2} f\right)$.
} 
up the gap in the chain of self-dual models found in [21] from $\mathcal{L}_{S D 1}^{(3)}$ to $\mathcal{L}_{S D 4}^{(3)}$. In the next section we investigate this issue by studying the particle content of the family of fourth order models (19).

\section{THE PARTICLE CONTENT OF $\mathcal{L}_{(a, b)}$}

A key ingredient in the master action approach is the fact that both the higher and the lower derivative terms have no particle content. It turns out that the fourth order term present in the fourth order self-dual model of [22] contains 2 d.o.f. and one of them is a ghost. This was an obstacle to go beyond the fourth order self-dual model in [22]. The fourth order term of [22] corresponds to $\mathcal{L}_{(a, b)}$ with $b=-a$ while the one we have used in (21) corresponds to $b=-3 a / 4$. The latter has no particle content. In order to clarify this issue and investigate any other possibility we examine the particle content of $\mathcal{L}_{(a, b)}$. As a byproduct we offer an alternative proof of absence of content in the $b=-3 a / 4$ case.

Explicitly, the $\mathcal{L}_{(a, b)}$ model (19) is given by

$$
\begin{aligned}
\mathcal{L}_{(a, b)}= & a h_{\mu \nu \rho} \square^{2} h^{\mu \nu \rho}+3 a \partial^{\mu} h_{\mu \nu \rho} \square \partial_{\lambda} h^{\lambda \nu \rho} \\
& +b\left(h_{\mu} \square^{2} h^{\mu}-2 \partial^{\mu} \partial^{\nu} h_{\mu \nu \alpha} \square h^{\alpha}\right) \\
& -\frac{(9 a+5 b)}{4} \partial \cdot h \square \partial \cdot h+(3 a+b)\left[\left(\partial^{\mu} \partial^{\nu} h_{\mu \nu \rho}\right)^{2}\right. \\
& \left.+\partial^{\mu} \partial^{\nu} \partial^{\rho} h_{\mu \nu \rho} \partial \cdot h\right] .
\end{aligned}
$$

Since the term $h_{\mu \nu \rho} \square^{2} h^{\mu \nu \rho}$ is required in order to have a truly spin-3 content we assume henceforth $a=1$ and rename the model as $\mathcal{L}_{b} \equiv \mathcal{L}_{(1, b)}$. It is invariant, for arbitrary values of $b$, by the traceless reparametrizations (17) determined by five gauge parameters $\bar{\Lambda}_{\mu \nu}$ which allow us to fix five gauge conditions.

Because of higher order time derivatives, the analysis of the particle content of the free theory $\mathcal{L}_{b}$ is nontrivial. Henceforth, we follow the approach of [28]; see also $[19,25]$. We first fix a gauge at action level, find a general solution of the gauge conditions in terms of helicity variables without introducing time derivatives, and plug it back in the action. Whenever we fix a gauge at action level we might lose equations of motion which may not be recovered from the remaining equations of motion. According to the recent references $[29,30]$ in order not to lose relevant equations of motion we are only allowed to fix at action level the so-called complete gauge conditions. In our case this means that our five gauge conditions must uniquely fix the five independent parameters $\bar{\Lambda}_{\mu \nu}$ without any freedom for integration constants. It can be shown that the five gauge conditions

$$
\partial_{j} h_{j k \mu}=0,
$$

where $j, k=1,2, \mu=0,1,2$, are complete. Namely, requiring that the conditions (38) are reached starting from arbitrary field configurations,

$$
\partial_{j}\left(h_{j k \mu}+\partial_{j} \bar{\Lambda}_{k \mu}+\partial_{k} \bar{\Lambda}_{j \mu}+\partial_{\mu} \bar{\Lambda}_{j k}\right)=0
$$

we uniquely determine the five parameters ${ }^{7} \bar{\Lambda}_{k \mu}$ by repeatedly applying space derivatives on (39). We obtain

$\bar{\Lambda}_{k \mu}=-\frac{1}{\nabla^{2}} \partial_{j} h_{j k \mu}+\frac{1}{\nabla^{4}} \partial_{(k} \partial_{i} \partial_{j} h_{i j \mu)}-\frac{\partial_{k} \partial_{\mu}}{3 \nabla^{6}}\left(\partial_{i} \partial_{j} \partial_{l} h_{i j l}\right)$.

The general solution to (38) is given in terms of five independent fields:

$$
\begin{array}{cc}
h_{j k l}=\hat{\partial}_{j} \hat{\partial}_{k} \hat{\partial}_{l} \psi ; & h_{j k 0}=\hat{\partial}_{j} \hat{\partial}_{k} \phi, \\
h_{00 j}=\hat{\partial}_{j} \gamma+\partial_{j} \Gamma ; & h_{000}=\rho,
\end{array}
$$

where $\hat{\partial}_{j}=\epsilon_{j k} \partial_{k}$ satisfies $\hat{\partial}_{i} \hat{\partial}_{j}=\nabla^{2} \delta_{i j}-\partial_{i} \partial_{j}$ and $\hat{\partial}_{i} \hat{\partial}_{i}=$ $\partial_{j} \partial_{j}=\nabla^{2}$. Back in (37) with $a=1$ we have the decoupling of the couple $(\gamma, \psi)$ from the trio $(\phi, \gamma, \rho)$,

$$
\mathcal{L}_{b}=\mathcal{L}_{\gamma \psi}+\mathcal{L}_{\phi \Gamma \rho},
$$

where

$$
\mathcal{L}_{\gamma \psi}=-(b+3) \gamma \nabla^{6} \gamma+2 b \gamma \nabla^{6} \square \psi-(b+1) \psi \nabla^{6} \square^{2} \psi,
$$

and

$$
\begin{aligned}
\mathcal{L}_{\phi \Gamma \rho}= & -\frac{(b+1)}{4}\left(\tilde{\rho} \square \square^{2} \tilde{\rho}+3 \tilde{\rho} \square \nabla^{2} \tilde{\rho}\right)-\frac{3}{2}(b+1) \Gamma \nabla^{2} \square \dot{\tilde{\rho}} \\
& -3(b+1) \tilde{\rho} \square \nabla^{4} \phi-(b+3) \tilde{\rho} \nabla^{6} \phi-(b+3) \phi \square \nabla^{6} \phi \\
& -3(b+3) \phi \nabla^{8} \phi+3(b+3) \phi \nabla^{6} \dot{\Gamma}-\frac{9}{4}(b+1) \Gamma \square \nabla^{4} \Gamma,
\end{aligned}
$$

where $\tilde{\rho}=\rho-3 \nabla^{2} \phi$ and $\dot{f}=\partial_{t} f$. If $b \neq-3$ we can write

$$
\mathcal{L}_{\gamma \psi}=-(b+3) \tilde{\gamma} \nabla^{6} \tilde{\gamma}-4 \frac{(b+3 / 4)}{(b+3)} \psi \nabla^{6} \square^{2} \psi,
$$

where $\tilde{\gamma} \equiv \gamma-(b /(b+3)) \square \psi$ decouples from $\psi$. Because of the double massless pole we have, in general, a massless ghost unless $b=-3 / 4$ where $\mathcal{L}_{\gamma \psi}$ has no particle content.

\footnotetext{
${ }^{7}$ Recall that $\eta^{\mu \nu} \bar{\Lambda}_{\mu \nu}=0$; therefore, $\bar{\Lambda}_{00}=\bar{\Lambda}_{j j}$ is not an independent variable.
} 
On the other hand, if $b=-3$, after the trivial shift $\gamma \rightarrow$ $\bar{\gamma}+(2 / 3) \square \psi$ in (46) we have

$$
\begin{aligned}
\left(\mathcal{L}_{\gamma \psi}\right)_{b=-3}= & -6 \bar{\gamma} \nabla^{6} \square \psi=-\frac{3}{2}\left[(\bar{\gamma}+\psi) \nabla^{6} \square(\bar{\gamma}+\psi)\right. \\
& \left.-(\bar{\gamma}-\psi) \nabla^{6} \square(\bar{\gamma}-\psi)\right] .
\end{aligned}
$$

Therefore, even if we change the overall sign of the Lagrangian, one of the two modes $\bar{\gamma} \pm \psi$ is a ghost. Independently of what we find in $\mathcal{L}_{\phi \Gamma \rho}$ we conclude that whenever $b \neq-3 / 4$ the Lagrangian $\mathcal{L}_{b}$ contains at least one ghost mode. This is in agreement with the analysis made in [22] for the case $b=-1$ which appears in the fourth order self-dual model of that reference. From now on we focus on the only possible ghost free case: $b=-3 / 4$. After the redefinition

$$
\Gamma=\tilde{\Gamma}-\dot{\tilde{\rho}} /\left(3 \nabla^{2}\right),
$$

we get rid of the highest time derivatives present in (45). We perform another redefinition

$$
\tilde{\rho}=\bar{\rho}+3 \nabla^{2} \phi,
$$

in order to cancel out all time derivatives of $\phi$. So we end up with

$$
\begin{aligned}
\mathcal{L}_{b=-\frac{3}{4}}= & -\frac{81}{4} \phi \nabla^{8} \phi-\frac{9}{2} \phi \nabla^{6} \bar{\rho}+\frac{27}{4} \phi \nabla^{6} \dot{\tilde{\Gamma}}-\frac{1}{4} \bar{\rho} \nabla^{2} \square \bar{\rho} \\
& -\frac{9}{16} \tilde{\Gamma} \nabla^{4} \square \tilde{\Gamma}=-\frac{81}{4} \bar{\phi} \nabla^{8} \bar{\phi}-\frac{9}{16} \bar{\Gamma} \nabla^{6} \bar{\Gamma},
\end{aligned}
$$

where

$$
\bar{\phi}=\phi-\frac{\dot{\tilde{\Gamma}}}{6 \nabla^{2}}+\frac{\bar{\rho}}{9 \nabla^{2}} ; \quad \bar{\Gamma}=\tilde{\Gamma}-\frac{2}{3 \nabla^{2}} \dot{\bar{\rho}}
$$

The equations of motion of (50) lead to trivial solutions $\bar{\phi}=0=\bar{\Gamma}$. Therefore, $\mathcal{L}_{b=-\frac{3}{4}}$ has no particle content. Since we have made several changes of variables involving time derivatives we should make sure that the two sets of fields $\Phi_{K}=(\phi, \Gamma, \rho)$ and $\bar{\Phi}_{K}=(\bar{\phi}, \bar{\Gamma}, \bar{\rho})$ are canonically equivalent. Notice that the diagonal form (50) could have been obtained at once from (45) at $b=-3 / 4$ via

$$
\begin{gathered}
\phi=\bar{\phi}+\frac{\dot{\bar{\Gamma}}}{9 \nabla^{2}}-\frac{\square}{9 \nabla^{4}} \bar{\rho}, \\
\Gamma=-\dot{\bar{\phi}}+\left(5+\frac{\square}{\nabla^{2}}\right) \frac{\bar{\Gamma}}{6}+\left(\frac{\square+3 \nabla^{2}}{9 \nabla^{2}}\right) \dot{\bar{\rho}}, \\
\rho=6 \nabla^{2} \bar{\phi}+\dot{\bar{\Gamma}}+\left(\frac{3 \nabla^{2}-2 \square}{3 \nabla^{4}}\right) \bar{\rho} .
\end{gathered}
$$

In matrix form we have $\Phi_{J}=\hat{M}_{J K} \bar{\Phi}_{K}$. The differential matrix operator $\hat{M}_{J K}$ can be read off from (52)-(54), it turns out remarkably that $\operatorname{det}(\hat{M})=1$. The reader can check explicitly that $\hat{M}_{J K} \Phi_{K}=0 \rightarrow \Phi_{k}=0$. Therefore, $\Phi_{J}$ and $\bar{\Phi}_{J}$ are indeed canonically equivalent. Moreover, the absence of $\bar{\rho}$ in (50) follows from a residual symmetry of the gauge (38) at $b=-3 / 4$. At this specific point a transverse Weyl symmetry shows up. The residual symmetry can be revealed by requiring invariance of the gauge (38) under $\delta h_{\mu \nu \rho}=\partial_{(\mu} \bar{\Lambda}_{\nu \rho)}+\eta_{(\mu \nu} \psi_{\rho)}^{T}$.

In summary, the fourth order Lagrangians $\mathcal{L}_{(a, b)}$ contain a ghost mode for all values of $b$ except $b=-3 a / 4$ where the model has no propagating d.o.f. Regarding the local symmetries, the traceless reparametrization can be enlarged in only two cases. We can have longitudinal Weyl symmetry $\delta h_{\mu \nu \rho}=\eta_{(\mu \nu} \partial_{\rho)} \lambda$ at $b=-a$ or transverse Weyl symmetry $\delta h_{\mu \nu \rho}=\eta_{(\mu \nu} \psi_{\rho)}^{T}$ at $b=-3 a / 4$. In the first case the six parameters $\left(\bar{\Lambda}_{\mu \nu}, \lambda\right)$ can be combined into arbitrary reparametrizations governed by a traceful tensor $\Lambda_{\mu \nu}$ which is, however, not sufficient to make the theory ghost-free as we have seen. In the second case we have maximal symmetry with seven independent parameters $\left(\bar{\Lambda}_{\mu \nu}, \psi_{\mu}^{T}\right)$ and we end up with no particle content.

Now we are ready to come back to investigate the existence of a possible fourth order self-dual model connected via some master action with our fifth order model (21). The natural candidate for the fourth order term is $\mathcal{L}_{b=-3 / 4}$ for two reasons: it is the lower derivative term in $\mathcal{L}_{S D 5}^{(3)}$ and it has no particle content. However, if we built up a fourth order self-dual model only in terms of a totally symmetric rank-3 tensor $h_{\mu \nu \rho}$, i.e., $\mathcal{L}_{S D 4}^{(3)}=\mathcal{L}_{(a, b)}\left(h_{\mu \nu \rho}\right)+\cdots$, where the dots stand for lower derivative terms, then the equations of motion $\delta S_{S D 4} / \delta h_{\mu \nu \rho}=0$ must be of the form

$$
E_{\mu}^{\beta} h_{\beta \nu \rho}^{*}(h)+E_{\nu}^{\beta} h_{\beta \rho \mu}^{*}(h)+E_{\rho}^{\beta} h_{\beta \mu \nu}^{*}(h)+\cdots=0,
$$

where $h_{\beta \nu \rho}^{*}(h)$ is of third order in derivatives and stem entirely from $\mathcal{L}_{(a, b)}\left(h_{\mu \nu \rho}\right)$. Since we only have a totally symmetric field $h_{\mu \nu \rho}$ by hypothesis, $h_{\beta \nu \rho}^{*}(h)$ must be also totally symmetric $h_{\beta \nu \rho}^{*}=h_{(\beta \nu \rho)}^{*}$. So if we apply $\partial^{\mu} \eta^{\nu \rho}$ on (55), the resulting equation vanishes identically except for lower derivative terms hidden in the dots. This means that the fourth order term $\mathcal{L}_{(a, b)}\left(h_{\mu \nu \rho}\right)$ present in $\mathcal{L}_{S D 4}^{(3)}$ must be invariant under longitudinal Weyl transformations ${ }^{8}$ :

$$
\delta_{\lambda} h_{\mu \nu \rho}=\eta_{(\mu \nu} \partial_{\rho)} \lambda .
$$

This is only possible if $b=-a$ which rules out the good candidate $b=-3 a / 4$. Therefore, a possible fourth order

$$
\overline{\delta_{\lambda} h_{\mu \nu \rho}^{8} d^{3} x \lambda}\left(\eta^{\mu \nu} \partial^{\rho}\right) \frac{\delta S_{S D 4}}{\delta h_{\mu \nu \rho}}=0=-\frac{1}{3} \int d^{3} x \frac{\delta S_{S D L}}{\delta h_{\mu \nu \rho}} \eta_{(\mu \nu} \partial_{\rho)} \lambda=\int d^{3} x \frac{\delta S_{D L 4}}{\delta h_{\mu \nu \rho}}
$$


self-dual model made out of the $b=-3 a / 4$-term must have auxiliary fields besides $h_{\mu \nu \alpha}$ in order to avoid the above argument. This is under investigation.

\section{CONCLUSION}

In $D=2+1$ dimensions, elementary particles of helicity +1 or -1 can be described either by the second order (in derivatives) Maxwell-Chern-Simons model of [10] or by the first order SD model of [31]. In the spin-2 case there are four (see [10,11,20] and [16,25]) self-dual models $\mathcal{L}_{S D j}^{(2)}$, $j=1,2,3,4$ describing +2 or -2 helicity particles via $j$ th order theories. For spin-3/2 we have three self-dual models of first, second [32,33], and third [18] order. In all those cases the $(j+1)$ th order model can be obtained from the $j$ th order one via master action and also via a NGE procedure. There seems to be a $2 s$ rule regarding the highest possible order for a spin- $s$ self-dual model without ghosts. There is however, a caveat in the spin-3 case.

In Sec. II we have revisited the derivation of the sixth order spin-3 self-dual model $\mathcal{L}_{S D 6}^{(3)}$ [19]. The model is made out of one sixth plus one fifth order term. One can first obtain the fifth order term based on Weyl and arbitrary reparametrization invariances; then the sixth order term is obtained via a symmetrized curl of the fifth order term.

Next, in order to go one step downward and derive $\mathcal{L}_{\text {SD5 }}^{(\dot{3})}$ we have started with the fifth order term of $\mathcal{L}_{S D 6}^{(3)}$ and searched for a convenient fourth order term. Since lower order requires less symmetry, we have obtained a fourth order term by requiring traceless reparametrization invariance (17) instead of general reparametrizations. This is also motivated by the key role of traceless reparametrizations in higher spin theories in $D=3+1$. In particular, this is the symmetry behind the massless limit of the massive spin-3 Singh-Hagen model [2]. This requirement leads to the family of fourth order terms $\mathcal{L}_{(a, b)}$ in (19). Imposing that the fifth order term is the symmetrized curl of the fourth order term uniquely determines $b=-3 a / 4$ where a transverse Weyl symmetry shows up. Consequently the new model $\mathcal{L}_{S D 5}^{(3)}$ is uniquely determined.

In Sec. III we have connected $\mathcal{L}_{S D 5}^{(3)}$ with $\mathcal{L}_{S D 6}^{(3)}$ via master action which guarantees that the particle content of $\mathcal{L}_{S D 5}^{(3)}$ is the same one of $\mathcal{L}_{S D 6}^{(3)}$, i.e., massive particles of helicity +3 or -3 without ghosts. In Sec. IV we have investigated the possibility of going another step downward $\mathcal{L}_{S D 5}^{(3)} \rightarrow \mathcal{L}_{S D 4}^{(3)}$.
A detailed study of $\mathcal{L}_{(a, b)}$ reveals that only the case $b=$ $-3 a / 4$ has no particle content and could be a good candidate to be the highest order term of a possible fourth order self-dual model $\mathcal{L}_{S D 4}^{(3)}$. However, we have argued that it cannot be entirely formulated in terms of a totally symmetric field $h_{\mu \nu \rho}$; auxiliary fields are required. We conjecture that only the self-dual models of order $2 s$ and $2 s-1$ can be formulated in terms of totally symmetric rank-s fields $h_{\alpha_{1} \ldots \alpha_{s}}$ without auxiliary fields. This is under investigation.

In summary, although we can obtain $\mathcal{L}_{S D 6}^{(3)}$ from the new fifth order model found here $\mathcal{L}_{S D 5}^{(3)}$ as well as the models $\mathcal{L}_{S D j}^{(3)}$, and $j=2,3,4$ can be obtained from $\mathcal{L}_{S D 1}^{(3)}$ of [20], there is no connection between those two sets of models. The key point is that the fourth order term of $\mathcal{L}_{S D 4}^{(3)}$ of [21] has a nontrivial particle content, so it cannot be used to produce an equivalent fifth order model via master action. From the point of view of local symmetries, the fourth order model of [21] is invariant under traceless reparametrizations plus longitudinal Weyl transformations $\delta h_{\mu \nu \rho}=$ $\partial_{(\mu} \bar{\Lambda}_{\nu \rho)}+\eta_{(\mu \nu} \partial_{\rho)} \Phi$ which is equivalent to full reparametrizations $\delta h_{\mu \nu \rho}=\partial_{(\mu} \Lambda_{\nu \rho)}$ while the model $\mathcal{L}_{S D 5}^{(3)}$ found here is invariant under traceless reparametrizations and transverse Weyl transformations $\delta h_{\mu \nu \rho}=\partial_{(\mu} \bar{\Lambda}_{\nu \rho)}+\eta_{(\mu \nu} \psi_{\rho)}^{T}$. So there is no way of connecting those theories via Noether gauge embedding.

We believe that if we could be able to go all the way downward from $\mathcal{L}_{S D 6}^{(3)}$ until a first order model, we would not end up at the model of [20]. There is probably a more natural first order spin-3 self-dual model which would allow us to go back upward until $\mathcal{L}_{S D 6}^{(3)}$. Eventually we might be able to construct first order self-dual models for arbitrary spin- $s$ in a more systematic way and learn more about higher spin theories.

\section{ACKNOWLEDGMENTS}

The work of D. D. is partially supported by CNPq (Grant No. 306380/2017-0). A. L. R. dos S. is supported by a CNPq-PDJ (Grant No. 150524/2018-8) while the work of R. R. L. dos S. has been supported by FAPESP Grants No. 2016/09489-0 and No. 2017/23966-9. D. D. thanks E. L. Mendonça for a discussion.
[1] M. Fierz, Helv. Phys. Acta 12, 3 (1939); M. Fierz and W. Pauli, Proc. R. Soc. A 173, 211 (1939).

[2] L. P. S. Singh and C. R. Hagen, Phys. Rev. D 9, 898 (1974).
[3] M. Stone, Illinois preprint, Report No. ILL-(TH)-89-23, 1989; Phys. Rev. Lett. 63, 731 (1989); Nucl. Phys. B327, 399 (1989). 
[4] R. Banerjee and S. Kumar, Phys. Rev. D 60, 085005 (1999).

[5] A. Ilha and C. Wotzasek, Phys. Rev. D 63, 105013 (2001).

[6] C. Wotzasek, arXiv:hep-th/9806005 (unpublished).

[7] D. Dalmazi, A. de Souza Dutra, and E. M. C. Abreu, Phys. Rev. D 74, 025015 (2006); 79, 109902(E) (2009).

[8] D. Dalmazi and E. L. Mendonça, Phys. Rev. D 80, 025017 (2009).

[9] D. Dalmazi and E. L. Mendonça, Phys. Rev. D 82, 105009 (2010).

[10] S. Deser, R. Jackiw, and S. Templeton, Ann. Phys. (N.Y.) 140, 372 (1982).

[11] S. Deser and J. McCarthy, Phys. Lett. B 246, 441 (1990).

[12] E. Bergshoeff, O. Hohm, and P. K. Townsend, Phys. Rev. Lett. 102, 201301 (2009).

[13] E. L. Mendonça, D. S. Lima, and A. L. R. dos Santos, Phys. Lett. B 783, 387 (2018).

[14] S. Deser and R. Jackiw, Phys. Lett. B 139, 371 (1984).

[15] D. Dalmazi and E. L. Mendonça, Phys. Rev. D 79, 045025 (2009).

[16] D. Dalmazi and E. L. Mendonça, J. High Energy Phys. 09 (2009) 011.

[17] M. A. Anacleto, A. Ilha, J. R. S. Nascimento, R. F. Ribeiro, and C. Wotzasek, Phys. Lett. B 504, 268 (2001).

[18] E. L. Mendonça, A. L. R. dos Santos, and D. S. Lima, Phys. Lett. B 775, 147 (2017).

[19] E. A. Bergshoeff, O. Hohm, and P. K. Townsend, Ann. Phys. (Amsterdam) 325, 1118 (2010).
[20] C. Aragone and A. Khoudeir, Rev. Mex. Fis. 39, 819 (1993), https://rmf.smf.mx/pdf/rmf/39/6/39_6_819.pdf.

[21] E. L. Mendonça and D. Dalmazi, Phys. Rev. D 91, 065037 (2015).

[22] D. Dalmazi and E. L. Mendonça, Eur. Phys. J. C 76, 373 (2016).

[23] C. Aragone and A. Khoudeir, Massive triadic Chern-Simons spin-3 theory, in Proceedings of the SILARG VII (World Scientific, Singapore, 1994), p. 529.

[24] T. Damour and S. Deser, Ann. Inst. Henri Poincaré, A, 47, 277 (1987).

[25] R. Andringa, E. A. Bergshoeff, M. de Roo, O. Hohm, E. Sezgin, and P. K. Townsend, Classical Quantum Gravity 27, 025010 (2010).

[26] D. Dalmazi and E. L.Mendonça, Phys. Rev. D 94, 025033 (2016).

[27] D. Dalmazi and A. L. R. dos Santos (to be published).

[28] S. Deser, Phys. Rev. Lett. 103, 101302 (2009).

[29] H. Motohashi, T. Suyama, and K. Takahashi, Phys. Rev. D 94, 124021 (2016).

[30] H. Motohashi, T. Suyama, and M. Yamaguchi, J. High Energy Phys. 06 (2018) 133.

[31] P. K. Townsend, K. Pilch, and P. van Nieuwenhuizen, Phys. Lett. 136B, 38 (1984).

[32] S. Deser and J. H. Kay, Phys. Lett. 120B, 97 (1983).

[33] S. Deser, Phys. Lett. 140B, 321 (1984). 